\title{
LEFT-INVARIANT MINIMAL UNIT VECTOR FIELDS ON A LIE GROUP OF CONSTANT NEGATIVE SECTIONAL CURVATURE
}

\author{
SEUnGHun Yi
}

\begin{abstract}
We find all left-invariant minimal unit vector fields and strongly normal unit vector fields on a Lie group which is isometric to the hyperbolic space.
\end{abstract}

\section{Introduction}

A smooth unit vector field on a Riemannian manifold $(M, g)$ is a cross section of its unit sphere bundle $T^{1}(M)$ and hence can be viewed as a submanifold of $T^{1}(M)$. If the manifold $M$ is compact and $T^{1}(M)$ is equipped with a natural Riemannian metric $g_{s}$ called the Sasaki metric, then the volume of the unit vector field is defined as the volume of this submanifold.

For the problem of determining unit vector fields which have minimal volume, Gluck and Ziller showed that the unit vector fields of minimal volume on $S^{3}$ are precisely the Hopf vector fields and no others ([7]). But in the higher dimensional spheres, $S^{2 n+1}, k \geq 2$, this is not the case ([4], [8], [10]).

The problem of finding unit vector fields of the minimum volume seems to be very difficult, so it is natural to consider the problem of finding the critical values or critical points of the volume functional.

Gil-Medriano and Llinares-Fuster proved that a unit vector field is a critical point of the volume functional if and only if the corresponding immersion in $\left(T^{1} M, g_{s}\right)$ is minimal ([3]). So we call such unit vector fields minimal even though the manifold is not compact.

Many examples of Riemannian manifolds and Lie groups equipped with leftinvariant minimal unit vector fields are provided ([1], [2], [3], [5], [6], [12], [13]). But there are very few manifolds on which we know all the minimal unit vector fields. Even for almost examples of the Lie groups, not all the left-invariant minimal unit vector fields are provided but only some of them are found to be minimal.

Received September 26, 2008.

2000 Mathematics Subject Classification. 53C20, 53C25, 53C42.

Key words and phrases. left-invariant minimal unit vector field, Lie group, hyperbolic manifold. 
The aim of this paper is to provide a Lie group with all the left-invariant minimal unit vector fields as follows. For an integer $n>1$, a Lie group $G_{n}$ is defined as follows.

$$
G_{n}:=\left\{\left(\begin{array}{cc}
1 & 0 \\
v & s I_{n-1}
\end{array}\right) \in G L(n, \mathbb{R}) \mid v \in \mathbb{R}^{n-1}, s>0\right\},
$$

where $v \in \mathbb{R}^{n-1}$ and $I_{n-1}$ is the $(n-1) \times(n-1)$ identity matrix. Then the Lie algebra $\mathfrak{g}_{n}$ of $G_{n}$ consists of the $n \times n$ matrices of the form

$$
\left(\begin{array}{cc}
0 & 0 \\
v & s I_{n-1}
\end{array}\right), v \in \mathbb{R}^{n-1}, s \in \mathbb{R} .
$$

Let $\left\{e_{1}, e_{2}, \ldots, e_{n-1}\right\}$ be the usual orthonormal basis for $\mathbb{R}^{n-1}$. Put

$$
E_{i}=\left(\begin{array}{cc}
0 & 0 \\
e_{i} & 0
\end{array}\right), i<n, \quad E_{n}=\left(\begin{array}{cc}
0 & 0 \\
0 & I_{n-1}
\end{array}\right) .
$$

Then the set $\left\{E_{1}, E_{2}, \ldots, E_{n-1}, E_{n}\right\}$ is an orthonormal bases for $\mathfrak{g}_{n}$.

The set of all left-invariant minimal unit vector fields on the Lie group $G_{n}$ is as follows which is the main result of this paper.

Theorem 1.1. For the Lie group $G_{2}$ every left-invariant unit vector field is minimal. For the Lie group $G_{n}, n>2$, the set of left-invariant minimal unit vector fields is $\left\{ \pm E_{n}\right\} \cup\left(\mathcal{S} \cap E_{n}^{\perp}\right)$, where $\mathcal{S}$ is the unit sphere of $\mathfrak{g}_{n}$.

In Section 2 we give some basic notions and facts. In Section 3 we prove the main theorem and in Section 4 we find the set of all strongly normal unit vector fields on the Lie group $G_{n}$.

The author would like to express his deep gratitude to K. B. Lee for many valuable discussions and to the anonymous referee for helpful comments.

\section{Minimal unit vector fields}

Let $(M, g)$ be a smooth Riemannian manifold, $\nabla$ be the Levi-Civita connection on $(M, g)$ and $R$ be the associated Riemannian curvature tensor with the sign convention $R_{X Y}=\nabla_{[X, Y]}-\left[\nabla_{X}, \nabla_{Y}\right]$.

We assume that the set $\chi^{1}(M)$ of unit vector fields on $M$ is non-empty. For $V \in \chi^{1}(M)$, let $L_{V}$ be a tensor field defined by

$$
L_{V}:=I+(\nabla V)^{*} \nabla V,
$$

where $I$ is the identity map and $(\nabla V)^{*}$ is the adjoint. Then $L_{V}$ is positive definite and symmetric. Put $f(V)=\left(\operatorname{det} L_{V}\right)^{\frac{1}{2}}$. For a closed manifold $M$, we define the volume functional $F: \chi^{1}(M) \longrightarrow \mathbb{R}$ by

$$
F(V):=\int_{M} f(V) d v
$$

where $d v$ is the volume form on $(M, g)$.

Now let $K_{V}$ be a $(1,1)$-tensor field define by

$$
K_{V}=f(V) \cdot L_{V}^{-1} \circ(\nabla V)^{*}
$$


and a 1-form $\omega_{V}$ associated to $V$ by

$$
\omega_{V}(X)=\operatorname{tr}\left(Z \longmapsto \nabla_{Z} K_{V}\right)(X) .
$$

For an orthonormal basis $\left\{E_{1}, E_{2}, \ldots, E_{n}\right\}$ of the tangent space, $\omega_{V}(X)$ is given as follows.

$$
\omega_{V}(X)=\sum_{i=1}^{n} g\left(\left(\nabla_{E_{i}} K_{V}\right)(X), E_{i}\right) .
$$

In [3] it is shown that a unit vector field $V$ is a minimal immersion if and only if the 1-form $\omega_{V}$ annihilates the distribution $\mathcal{H}^{V}$ consisting of tangent vectors orthogonal to $V$. Moreover it is shown that $V$ is a critical point of the volume functional if and only if the map $V: M \longrightarrow\left(T^{1} M, g_{s}\right)$ is a minimal immersion, where $\left(T^{1} M, g_{s}\right)$ is the unit tangent bundle $T^{1} M$ equipped with the Sasaki metric $g_{s}$. For this reason we define minimal unit vector fields on a manifold which is not necessarily compact as follows.

Definition 2.1. A unit vector field $\mathrm{V}$ on a Riemannian manifold $(M, g)$ is called minimal if $\omega_{V}(X)=0$ for all $X \in \mathcal{H}^{V}$.

From now on we consider left-invariant unit vector fields on Lie groups. Let $G$ be an $n$-dimensional connected Lie group equipped with a left-invariant metric and $\mathfrak{g}$ be its Lie algebra. Let $\mathcal{S}$ be the unit sphere of $\mathfrak{g}$ with respect to the inner product $\langle$,$\rangle which is determined by the left-invariant metric on G$. Since $V \in \mathcal{S}, \nabla V, L_{V}, K_{V}$ and $\omega_{V}$ are invariant by left translation, the function $f$ can be considered as a function on $\mathcal{S}$.

The distribution $\mathcal{H}^{V}$ is invariant by left translation and can be identified with the orthogonal complement $V^{\perp}$ of $V$ in $\mathfrak{g}$ and thus $V^{\perp}$ may be naturally identified with the tangent space $T_{V} \mathcal{S}$ of the unit sphere $\mathcal{S}$ at $V$. Thus a left-invariant unit vector field $V$ is minimal if and only if the 1-form $\omega_{V}$ on $\mathfrak{g}$ vanishes on $V^{\perp} \cong T_{V} \mathcal{S}([13])$.

Proposition 2.1 ([13], Proposition 2.1). For $X \in T_{V} \mathcal{S}$ we have

$$
\omega_{V}(X)=-d f_{V}(X)-\operatorname{tr} a d_{K_{V}} X
$$

and $V$ is minimal if and only if

$$
d f_{V}(X)=-\operatorname{tr} a d_{K_{V} X}
$$

for all $X \in T_{V} \mathcal{S}$.

Thus on a unimodular Lie group $G$, i.e., $\operatorname{tr} a d_{X}=0$, for all $X \in \mathfrak{g}$, a leftinvariant unit vector field $V$ is minimal if and only if $V$ is a critical point of the function $f$ on $\mathcal{S}$.

For a non-unimodular Lie group $G$ with a left-invariant metric, we denote by $\mathcal{U}$ its unimodular kernel, i.e.,

$$
\mathcal{U}=\left\{X \in \mathfrak{g} \mid \operatorname{tr} a d_{X}=0\right\} .
$$


Then $\mathcal{U}$ is an ideal of codimension 1 since $\operatorname{tr} a d_{X}$ is a linear functional. For a unit vector $H$ which is orthogonal to $\mathcal{U}$, the linear transformation $a d_{H}$ restricted to $\mathcal{U}$ is a derivation of $\mathcal{U}$. So we have the following.

Proposition 2.2 ([13], Proposition 2.5). Let $\mathcal{U}$ be the unimodular kernel of a non-unimodular Lie group such that $\left.a d_{H}\right|_{\mathcal{U}}$ is a symmetric endomorphism of $\mathcal{U}$ with respect to $\langle$,$\rangle . Then a left-invariant unit vector field V$ is minimal if and only if it is a critical point of the function $f$ on $\mathcal{S}$.

We shall use this proposition 2.2 to find all the left-invariant minimal unit vector fields on the Lie group $G_{n}$ which is isometric to the hyperbolic space $\mathbb{H}^{n}$.

\section{Proof of the main theorem}

For an integer $n>1$, define a Lie group $G_{n}$ as follows.

$$
G_{n}:=\left\{\left(\begin{array}{cc}
1 & 0 \\
v & s I_{n-1}
\end{array}\right) \in G L(n, \mathbb{R}) \mid v \in \mathbb{R}^{n-1}, s>0\right\},
$$

where $v \in \mathbb{R}^{n-1}$ and $I_{n-1}$ is the $(n-1) \times(n-1)$ identity matrix. Then the Lie algebra $\mathfrak{g}_{n}$ of $G_{n}$ consists of the $n \times n$ matrices of the form

$$
\left(\begin{array}{cc}
0 & 0 \\
v & s I_{n-1}
\end{array}\right), v \in \mathbb{R}^{n-1}, s \in \mathbb{R} \text {. }
$$

Let $\left\{e_{1}, e_{2}, \ldots, e_{n-1}\right\}$ be the usual orthonormal basis for $\mathbb{R}^{n-1}$. Put

$$
E_{i}=\left(\begin{array}{cc}
0 & 0 \\
e_{i} & 0
\end{array}\right), i<n, \quad E_{n}=\left(\begin{array}{cc}
0 & 0 \\
0 & I_{n-1}
\end{array}\right) .
$$

Then we have $\left[E_{i}, E_{j}\right]=0$ for $1 \leq i, j<n$, and $\left[E_{n}, E_{k}\right]=E_{k}$ for $1 \leq k \leq n$.

Let $G_{n}$ be equipped with a left-invariant metric such that $\left\{E_{1}, E_{2}, \ldots, E_{n}\right\}$ is an orthonormal basis for $\mathfrak{g}_{n}$.

Let $\nabla$ be the Levi-Civita connection of $G_{n}$. Then for $X, Y, Z \in \mathfrak{g}$ it satisfies the following identity $([9])$.

$$
\left\langle\nabla_{X} Y, Z\right\rangle=\frac{1}{2}\{\langle[X, Y], Z\rangle-\langle[Y, Z], X\rangle+\langle[Z, X], Y\rangle\} .
$$

For $1 \leq i, j<n, 1 \leq k \leq n$, it is easy to show that

$$
\nabla_{E_{i}} E_{j}=\delta_{i j} E_{n}, \quad \nabla_{E_{i}} E_{n}=-E_{i}, \quad \nabla_{E_{n}} E_{k}=0 .
$$

Thus $K_{E_{i} \wedge E_{j}}=\left\langle R\left(E_{i}, E_{j}\right) E_{i}, E_{j}\right\rangle=-1$ and the Lie group $\left(G_{n},\langle\rangle,\right)$ has constant negative sectional curvature -1 . In fact it is simply connected and complete. Thus the Lie group $\left(G_{n},\langle\rangle,\right)$ is isometric to the hyperbolic space $H^{n}([11])$.

Proof of the Theorem 1.1. For $X=\sum_{i=1}^{n} a_{i} E_{i}$, we have

$$
\operatorname{tr} a d_{X}=\sum_{j=1}^{n}\left\langle a d_{X} E_{j}, E_{j}\right\rangle=n a_{n}
$$


So the unimodular kernel $\mathcal{U}$ is the set $\left\{X \in \mathfrak{g} \mid X=\sum_{i=1}^{n-1} a_{i} E_{i}\right\}$ and a unit vector orthogonal to $\mathcal{U}$ is $E_{n}$.

And for an element $X \in \mathcal{U}$ we have $a d_{H}(X)=X$ and thus $\left.a d_{H}\right|_{\mathcal{U}}=\left.I d\right|_{\mathcal{U}}$. Therefore $a d_{H} \mid \mathcal{U}$ is a symmetric endomorphism of $\mathcal{U}$ with respect to $\langle$,$\rangle . So by$ the Proposition 2.2, a left-invariant unit vector field $V$ is minimal if and only if $V$ is a critical point of $f$ on $\mathcal{S}$.

Let $W=\sum_{i=1}^{n-1} z_{i} E_{i}$ with $\sum_{i=1}^{n-1} z_{i}^{2}=1$ and $V=x E_{n}+y W$ with $x^{2}+y^{2}=1$. Then for $j<n, \nabla_{E_{j}} V=y z_{j} E_{n}-x E_{j}$ and $\nabla_{E_{n}} V=0$. So we have

$$
\nabla V=\sum_{i=1}^{n-1}\left(y z_{j} \otimes \alpha_{j}-x E_{j} \otimes \alpha_{j}\right),
$$

where $\left\{\alpha_{1}, \alpha_{2}, \ldots, \alpha_{n}\right\}$ is the dual coframe field of $\left\{E_{1}, E_{2}, \ldots, E_{n}\right\}$. The matrix form of $\nabla V$ is as follows.

So we have

$$
\nabla V=\left(\begin{array}{ccccc}
-x & 0 & \cdots & 0 & 0 \\
0 & -x & \cdots & 0 & 0 \\
\cdots & \cdots & \cdots & \cdots & \cdots \\
0 & 0 & \cdots & -x & 0 \\
y z_{1} & y z_{2} & \cdots & y z_{n-1} & 0
\end{array}\right)
$$

$$
f(V)=\sqrt{\operatorname{det}\left(I+(\nabla V)^{*} \nabla V\right)}=\sqrt{2}\left(1+x^{2}\right)^{\frac{n-2}{2}} .
$$

If $n=2$, the function $f$ becomes a constant function and thus every leftinvariant unit vector field is minimal.

Assume that $n>2$ and let

$$
\begin{aligned}
f\left(x, y, z_{1}, \ldots, z_{n-1}\right) & =\left(1+x^{2}\right)^{r} \\
g_{1}\left(x, y, z_{1}, \ldots, z_{n-1}\right) & =x^{2}+y^{2}-1=0 \\
g_{2}\left(x, y, z_{1}, \ldots, z_{n-1}\right) & =z_{1}^{2}+\cdots+z_{n-1}^{2}-1=0,
\end{aligned}
$$

where $r=\frac{n-2}{2}$.

By the Lagrange multiplier method, we have to solve the following simultaneous equation.

$$
\left\{\begin{array}{l}
\nabla f=\lambda \nabla g_{1}+\mu \nabla g_{2} \\
g_{1}=0 \\
g_{2}=0
\end{array}\right.
$$

Since

$$
\begin{aligned}
\nabla f & =\left(2 r x\left(1+x^{2}\right)^{r-1}, 0, \ldots, 0\right), \\
\nabla g_{1} & =(2 x, 2 y, 0, \ldots, 0), \\
\nabla g_{2} & =\left(0,0,2 z_{1}, \ldots, 2 z_{n-1}\right),
\end{aligned}
$$

the above equation becomes as follows:

$$
\left(2 r x\left(1+x^{2}\right)^{r-1}, 0, \ldots, 0\right)=\left(2 \lambda x, 2 \lambda y, 2 \mu z_{1}, \ldots, 2 \mu z_{n-1}\right) .
$$


So we have the following:

$$
\begin{aligned}
2 \lambda x & =2 r x\left(1+x^{2}\right)^{r-1}, \\
2 \lambda y & =0, \\
2 \mu z_{i} & =0, \quad i=1, \ldots, n-1, \\
x^{2}+y^{2} & =1, \\
z_{1}^{2}+\cdots+z_{n-1}^{2} & =1 .
\end{aligned}
$$

In the equation (5), if $\mu \neq 0$, then we have $z_{i}=0, i=1, \ldots, n-1$, and it contradicts to the equation (7). So we have $\mu=0$,

In (4), we have $\lambda=0$ or $y=0$.

(i) If $\lambda=0$, then $x=0$ and thus $y= \pm 1$.

(ii) If $\lambda \neq 0$, then $y=0$ and thus $x= \pm 1, \lambda=2 r$.

Therefore the set of critical points of $f$ are as follows.

$$
\left\{\left( \pm 1,0, z_{1}, \ldots, z_{n-1}\right),\left(0, \pm 1, z_{1}, \ldots, z_{n-1}\right)\right\}
$$

where $z_{1}, \ldots, z_{n-1}$ are arbitrary real numbers which satisfies the equation (5). This completes the proof.

\section{Strongly normal unit vectors}

A unit vector field $V$ on a Riemannian manifold $(M, g)$ is called normal if $R_{X Y}$ preserves $\mathcal{H}^{V}$ for all $X, Y \in \mathcal{H}^{V}$, i.e., $g\left(R_{X Y} Z, V\right)=0$ for all $X, Y, Z \in$ $\mathcal{H}^{V}$. And $V \in \chi^{1}(M, g)$ is called strongly normal if $g\left(\left(\nabla_{X} A_{V}\right) Y, Z\right)=0$ for all $X, Y, Z \in \mathcal{H}^{V}$. Here, $A_{V}=-\nabla V$. A vector field $V$ is called killing if $A_{V}$ is skew-symmetric. It is easy to see that strongly normal unit vector field is normal and a unit Killing vector field is strongly normal if and only if normal.

The set of all strongly normal unit vector fields on $G_{n}$ is given as follows.

Theorem 4.1. For $n \geq 2$, the set of all strongly normal unit vector fields on the Lie group $G_{n}$ is $\left\{ \pm E_{n}\right\} \cup\left(\mathcal{S} \cap E_{n}^{\perp}\right)$, where $\mathcal{S}$ is the unit sphere of $\mathfrak{g}_{n}$.

Proof. Let $X=\sum_{i=1}^{n} a_{i} E_{i}, Y=\sum_{j=1}^{n} b_{j} E_{j}, Z=\sum_{k=1}^{n} a_{k} E_{k}$, and $V=$ $\sum_{l=1}^{n} p_{l} E_{l}$. And assume that $a_{i}, b_{j}, c_{k}, p_{l}$ satisfies the conditions

$$
\|X\|=\|Y\|=\|Z\|=\|V\|=1
$$

and

$$
\langle X, V\rangle=\langle Y, V\rangle=\langle Z, V\rangle=0 .
$$


Then

$$
\begin{aligned}
\left(\nabla_{X} A_{V}\right)(Y) & =-\nabla_{X}\left(\nabla_{Y} V\right)+\nabla_{\nabla_{X} Y} V \\
& =\sum_{i=1}^{n-1}\left(-a_{i} b_{n} p_{i}+a_{i} b_{i} p_{n}\right) E_{n} \\
& =\left(\sum_{i=1}^{n} a_{i} b_{i}\right) p_{n} E_{n} .
\end{aligned}
$$

So we have

$$
\left\langle\left(\nabla_{X} A_{V}\right)(Y), Z\right\rangle=\left(\sum_{i=1}^{n} a_{i} b_{i}\right) c_{n} p_{n} .
$$

If $p_{n}=0$, then $V$ is strongly normal. Thus every vector in the set $\mathcal{S} \cap E_{n}^{\perp}$ is strongly normal. If $p_{1}=p_{2}=\cdots=p_{n-1}=0$, then $a_{n}=b_{n}=c_{n}=0$ and thus $E_{n}$ is strongly normal.

Now assume that $p_{n} \cdot p_{i} \neq 0$, for some $i, 1 \leq i<n$. Put

$$
X=Y=Z=\frac{1}{\sqrt{p_{i}^{2}+p_{n}^{2}}}\left(-p_{n} E_{i}+p_{i} E_{n}\right) .
$$

Then $\left\langle\left(\nabla_{X} A_{V}\right)(Y), Z\right\rangle=p_{n} \cdot p_{i} \neq 0$. So the vector $V=\sum_{i=1}^{n} p_{i} E_{l}$ with $p_{n} \cdot p_{i} \neq 0,1 \leq i<n$, is not strongly normal. This completes the proof.

By the Theorem 1.1 and the Theorem 4.1, for $n>2$, the set of minimal unit vector fields and the set of strongly normal unit vector fields on the Lie group $G_{n}$ are the same.

\section{References}

[1] E. Boeckx and L. Vanhecke, Harmonic and minimal radial vector fields, Acta Math. Hungar. 90 (2001), no. 4, 317-331.

[2] __ Harmonic and minimal vector fields on tangent and unit tangent bundles, Differential Geom. Appl. 13 (2000), no. 1, 77-93.

[3] O. Gil-Medriano and E. Llinares-Fuster, Minimal unit vector fields, Tohoku Math. J. (2) $\mathbf{5 4}$ (2002), no. 1, 71-84.

[4] Second variation of volume and energy of vector fields. Stability of Hopf vector fields, Math. Ann. 320 (2001), no. 3, 531-545.

[5] J. C. Gonzalez-Davila and L. Vanhecke, Examples of minimal unit vector fields, Ann. Global Anal. Geom. 18 (2000), no. 3-4, 385-404.

[6] __ Minimal and harmonic characteristic vector fields on three-dimensional contact metric manifolds, J. Geom. 72 (2001), no. 1-2, 65-76.

[7] H. Gluck and W. Ziller, On the volume of a unit vector field on the three-sphere, Comment. Math. Helv. 61 (1986), no. 2, 177-192.

[8] D. L. Johnson, Volumes of flows, Proc. Amer. Math. Soc. 104 (1988), no. 3, 923-931.

[9] J. Milnor, Curvatures of left invariant metrics on Lie groups, Advances in Math. 21 (1976), no. 3, 293-329.

[10] S. L. Pedersen, Volumes of vector fields on spheres, Trans. Amer. Math. Soc. 336 (1993), no. $1,69-78$.

[11] W. A. Poor, Differential Geometric Structures, McGraw-Hill Book Co., New York, 1981. 
[12] M. Salvai, On the volume of unit vector fields on a compact semi-simple lie group, Journal of Lie Theory 13 (2003), 455-462.

[13] K. Tsukada and L. Vanhecke, Invariant minimal unit vector fields on Lie groups, Period. Math. Hungar. 40 (2000), no. 2, 123-133.

Sciences and Liberal Arts (Mathematics)

YOUNGDONG UNIVERSITY

Chungbuk 370-701, Korea

E-mail address: seunghun@youngdong.ac.kr 